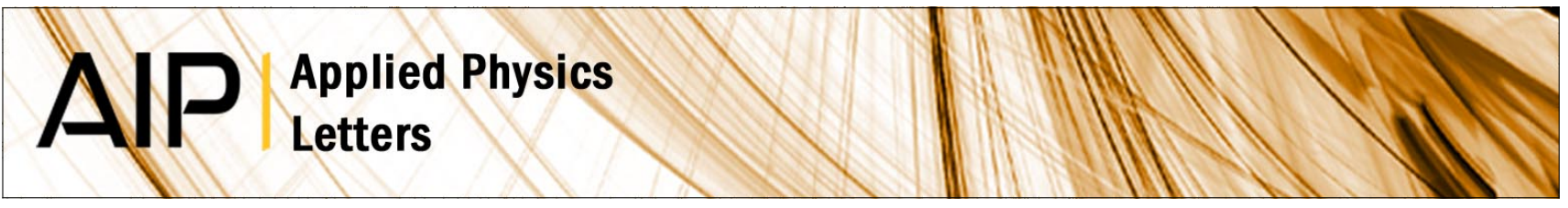

\title{
Superconductivity in heavily compensated Mg-doped InN
}

E. Tiras, M. Gunes, N. Balkan, R. Airey, and W. J. Schaff

Citation: Appl. Phys. Lett. 94, 142108 (2009); doi: 10.1063/1.3116120

View online: http://dx.doi.org/10.1063/1.3116120

View Table of Contents: http://apl.aip.org/resource/1/APPLAB/v94/i14

Published by the American Institute of Physics.

\section{Related Articles}

Water-induced two-dimensional Kondo-like scattering in $\mathrm{Na} 0.23 \mathrm{CoO} 2$ single crystals J. Appl. Phys. 113, 013703 (2013)

Spin and charge zero-bias conductance peak in a graphene-based Fd junction J. Appl. Phys. 112, 113910 (2012)

Consolidation of $\mathrm{Bi}-2223$ superconducting powders by spark plasma sintering J. Appl. Phys. 112, 113906 (2012)

Pressure effects on the superconducting thin film Ba1-xKxFe2As2

Appl. Phys. Lett. 101, 042601 (2012)

Pressure-induced positive electrical resistivity coefficient in Ni-Nb-Zr-H glassy alloy

Appl. Phys. Lett. 100, 253114 (2012)

\section{Additional information on Appl. Phys. Lett.}

Journal Homepage: http://apl.aip.org/

Journal Information: http://apl.aip.org/about/about_the_journal

Top downloads: http://apl.aip.org/features/most_downloaded

Information for Authors: http://apl.aip.org/authors

\section{ADVERTISEMENT} \section{AIP $\begin{aligned} & \text { Applied Physics } \\ & \text { Letters }\end{aligned}$}

\section{EXPLORE WHAT'S NEW IN APL}

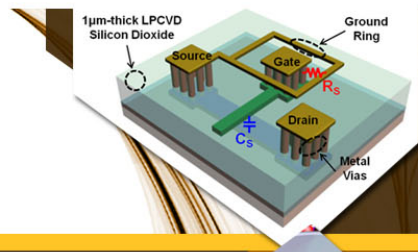

SURFACES AND INTERFACES

Focusing on physical, chemical, biological, structural, optical, magnetic and electrical properties of surfaces and interfaces, and more.. 


\title{
Superconductivity in heavily compensated Mg-doped InN
}

\author{
E. Tiras, ${ }^{1,2}$ M. Gunes, ${ }^{1}$ N. Balkan, ${ }^{1, a)}$ R. Airey, ${ }^{3}$ and W. J. Schaff ${ }^{4}$ \\ ${ }^{1}$ School of Computer Science and Electronic Engineering, University of Essex, Wivenhoe Park, \\ Colchester CO4 3SQ, United Kingdom \\ ${ }^{2}$ Department of Physics, Faculty of Science, Anadolu University, Yunus Emre Campus, \\ Eskisehir 26470, Turkey \\ ${ }^{3}$ Department of Electronic and Electrical Engineering, University of Sheffield, Mappin Street, \\ Sheffield S3 $7 H Q$, United Kingdom \\ ${ }^{4}$ Department of Electrical and Computer Engineering, Cornell University, Ithaca, New York 14853, USA
}

(Received 10 February 2009; accepted 11 March 2009; published online 10 April 2009)

\begin{abstract}
We report superconductivity in Mg-doped $\mathrm{InN}$ grown by molecular beam epitaxy. Superconductivity phase transition temperature occurs $T_{c}=3.97 \mathrm{~K}$ as determined by magnetoresistance and Hall resistance measurements. The two-dimensional (2D) carrier density of the measured sample is $n_{2 \mathrm{D}}=9 \times 10^{14} \mathrm{~cm}^{-2}$ corresponding to a three-dimensional (3D) electron density of $n_{3 \mathrm{D}}=1.8 \times 10^{19} \mathrm{~cm}^{-3}$ which is within the range of values between Mott transition and the superconductivity to metal transition. We propose a plausible mechanism to explain the existence of the superconductivity in terms of a uniform distribution of superconducting $\mathrm{InN}$ nanoparticles or nanosized indium dots forming microscopic Josephson junctions in the heavily compensated insulating bulk InN matrix. (C) 2009 American Institute of Physics. [DOI: 10.1063/1.3116120]
\end{abstract}

Until about a couple of decades ago the commonly accepted value for the InN band gap energy was $1.89 \mathrm{eV}$. However, the recent progress in epitaxial growth techniques has led to the availability of high quality $\mathrm{InN}$ yielding strong experimental evidence to show that the real band gap energy of $\mathrm{InN}$ is between 0.7 and $0.8 \mathrm{eV}$ at room temperature. ${ }^{2-4}$ This value is close to recent theoretical calculations. ${ }^{5}$ This observation had two important implications: first, many theoretical studies and experimental explanations based on the previously reported $\mathrm{InN}$ gap of $1.89 \mathrm{eV}$ had to be revised; second, as the wurtzite structured $\mathrm{InN}$ forms an alloy with $\mathrm{GaN}$ it became possible to form indium-rich ternary GaInN. Indium-rich $\mathrm{GaInN}$ can be utilized in optoelectronic device applications extending the well-known ultraviolet and blue spectral region applications of the Ga rich material to the near infrared, thus covering wavelengths from 200 to 1650 $\mathrm{nm}$ using a single material system. ${ }^{6-11}$

There is little experimental data on the electrical properties of InN particularly at low temperatures. Recently, Inushima et al. ${ }^{12}$ has reported the observation of superconducting behavior of InN. This is followed by other reports from the same group ${ }^{13-18}$ including the measurements of superconductivity transition temperature as a function of carrier density. A range of possible mechanisms to explain the transition has been explored by various research groups. Inushima et al. ${ }^{17}$ proposed that superconductivity is related to the presence of the In-In chains of finite length spread in the $a b$-plane. Recently it was reported that Si-doped $\mathrm{InN}$ with the carrier density between $2 \times 10^{18}$ and $1.5 \times 10^{19} \mathrm{~cm}^{-3}$ did not show superconductivity. ${ }^{18}$ This inhibition of superconductivity was interpreted in terms of the range of carrier densities imposing a limit on the transition. However, this range of carrier densities was challenged by Inushima et $a l .,{ }^{17}$ where it was reported that the lowest carrier density is limited by the Mott transition which is about $2 \times 10^{17} \mathrm{~cm}^{-3}$,

\footnotetext{
${ }^{\text {a) }}$ Author to whom correspondence should be addressed. Electronic mail: balkan@essex.ac.uk.
}

and the highest density is limited by the superconductivity to metal transition at about $7 \times 10^{20} \mathrm{~cm}^{-3}$.

Ling et al. ${ }^{19}$ reported superconductivity behavior in metal organic vapor phase epitaxy (MOVPE) grown InN samples supporting the interpretation of Inushima et al. ${ }^{17}$ that the superconductivity was due to the presence of In-In chains of finite length in the $a b$-plane. More recently, Kadir et $a .^{20}$ proposed an alternative mechanism in MOVPE grown material where they claimed the anisotropic and type II superconductivity to be associated with the presence of $\mathrm{In}_{2} \mathrm{O}_{3}$ impurities formed during the annealing process. ${ }^{20}$ $\mathrm{X}$-ray diffraction data indicated that the observed $\mathrm{In}_{2} \mathrm{O}_{3} \mathrm{im}$ purities may be the reason for the phase transition as indium oxide is a well-known superconductor at $T_{c}=3.2 \mathrm{~K}$ in granular and amorphous form. ${ }^{20}$ They reported superconductive transition at temperatures between $T_{c}=3.1$ and $0.5 \mathrm{~K}$ depending on the indium oxide concentration.

No consensus have, however, emerged to date about underlying physical mechanism for superconductivity in InN and it is worth pointing out that no observation of superconductivity has been reported in either In-rich GaInN or $\mathrm{Mg}$ doped InN. Present work presented here is concerned with the study of superconductivity in Mg-doped heavily compensated $\mathrm{InN}$.

The Mg-doped InN films were grown by a Varian GEN-II gas-source molecular beam epitaxy (MBE) on c-sapphire substrates with a roughly $200 \mathrm{~nm}$ thick GaN buffer layer. ${ }^{21}$ The thickness of the InN layer as determined from growth parameters and verified by backscattering spectrometry was nearly $500 \mathrm{~nm}$. The $\mathrm{Mg}$ concentration, as measured by secondary ion mass spectroscopy, ranged from $2 \times 10^{20}$ to $1 \times 10^{21} \mathrm{~cm}^{-3}$. A nominally undoped $\mathrm{InN}$ film was used as a standard for comparison; it was a $2700 \mathrm{~nm}$ thick InN film with a $300 \mathrm{~nm}$ thick GaN buffer layer. ${ }^{22}$ The Mg-doped InN (GS1548) and undoped InN (GS1382) samples were fabricated in Hall-bar geometry and Ohmic contacts were formed by diffusing the $\mathrm{Au} / \mathrm{Ni}$ alloy. At low temperatures the two-dimensional (2D) electron densities 

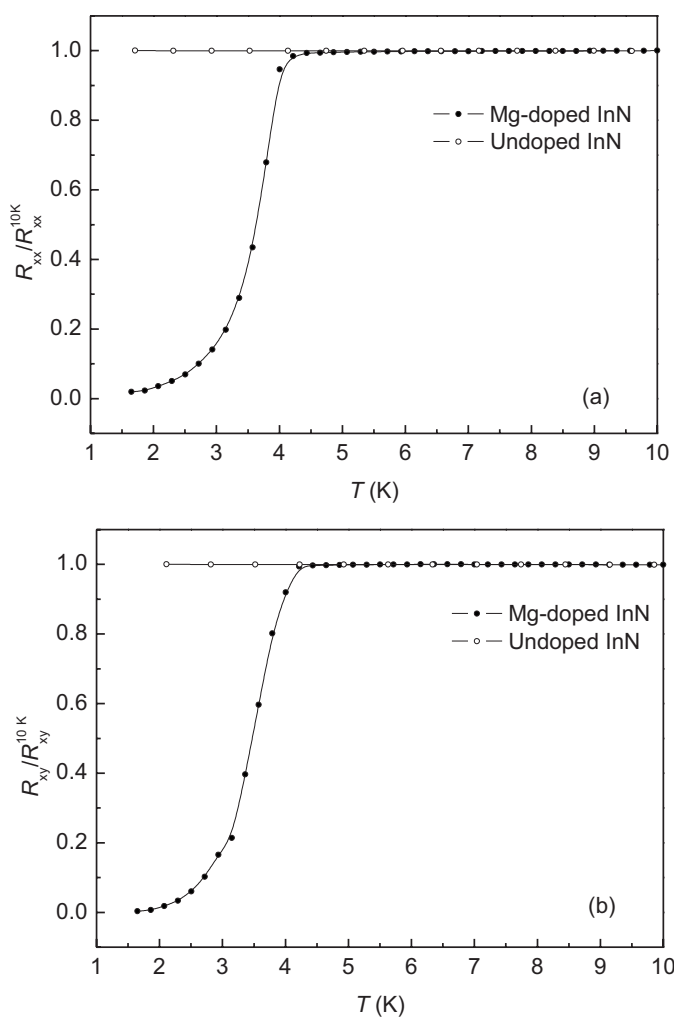

FIG. 1. The temperature dependence of the (a) $R_{x x}$ and (b) $R_{x y}$ both normalized with respect to their $10 \mathrm{~K}$ value for $\mathrm{Mg}$-doped and undoped $\mathrm{InN}$ samples.

were $n_{2 \mathrm{D}}=1.3 \times 10^{14}$ and $9 \times 10^{14} \mathrm{~cm}^{-2}$ in the undoped and Mg-doped material, respectively.

The resistance $\left(R_{x x}\right)$ along the applied current and Hall resistance $\left(R_{x y}\right)$ measurements were carried out as a function of (i) temperature at fixed magnetic and electric fields where the electric field was kept low enough to ensure Ohmic conditions, hence to avoid carrier heating and, (ii) as a function of magnetic field at a fixed electric field and at the lowest of the temperature range $(T \sim 1.6 \mathrm{~K})$. The $R_{x x}$ and $R_{x y}$ measurements were performed in a four terminal configuration in a cryogen free superconducting magnet system.

In this study, the dramatic reduction in the electrical resistivity, within about $1 \mathrm{~K}$ temperature interval, from 246 to $4.7 \Omega$ for $R_{x x}$ are attributed to transition to superconducting state (where the latter figure includes the lead resistances). Phase transition from the semiconducting state to superconducting state occurs at $T \leq 3.97 \mathrm{~K}$.

In Fig. 1 we show the sample resistance in a direction parallel and perpendicular to the current flow for both an undoped InN with a very high electron density and in $\mathrm{Mg}$ doped InN. It is clear from the figure that there is no evidence of superconductivity in the undoped (heavily $n$-type) material while the Mg-doped material exhibits superconductivity.

It has been claimed generally in the literature that the superconductivity in InN is anisotropic and type II..$^{12-17,19}$ Our sample also showed an anisotropic superconductivity. Figure 2 shows the clear evidence of the anisotropy in the upper critical magnetic field parallel and perpendicular to the $c$-axis. The angle-dependent threshold applied field values at which transitions occur are $H_{c 1}$ and $H_{c 2}{ }^{14}$ Due to the limitations in our experimental setup we could not obtain $H_{c 1}$. However $H_{c 2}$ values are 0.3410 and $1.2 \mathrm{~T}$ for the $B \| c$-axis,

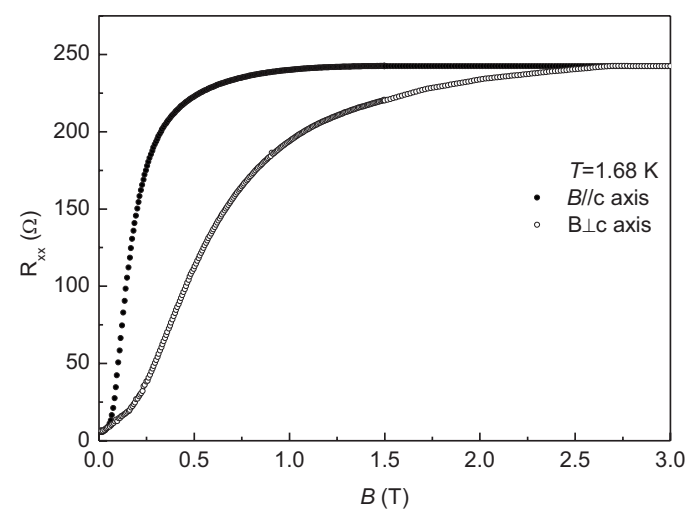

FIG. 2. Magnetic field dependence of $R_{x x}$ at $T=1.68 \mathrm{~K}$.

and $B \perp c$-axis, respectively. These values are much greater than the critical magnetic field of In which is $0.023 \mathrm{~T} .{ }^{11}$ This observation alone may suggest that it is bulk $\mathrm{InN}$ rather than In dots/chains that become superconductive. If it is $\mathrm{InN}$ becoming superconducting two equally plausible mechanisms may explain the observed behavior. First the metallic $2 \mathrm{D} \mathrm{InN}$ with a large density of electron accumulation at the surface $^{9-11}$ may, at low temperatures, become a 2D superconducting channel shorting completely the bulk conduction. If this is the case we should also be able to observe the semiconductor-superconductor transition in undoped InN. As we have shown in Fig. 1, there is no evidence for superconductivity in the undoped $\mathrm{InN}$ down to our lowest measurement temperatures of $T \leq 1.68 \mathrm{~K}$. The second possibility is the existence of either indium or metallic $\mathrm{InN}$ nanoparticles (quantum dots?) distributed in the heavily compensated InN matrix in the bulk of the material. In this case when the temperature is low enough for the heavily compensated $\mathrm{InN}$ to become an insulator and at the same time InN or In quantum dots to become superconducting we may then expect the formation of microscopic Josephson junctions that are distributed in the bulk of the sample. The transport then occurs via the superconducting tunneling at low enough applied voltages, and this shorts out the 2D conductivity at the surface. Whether the quantum dots (nanoparticles) are indium or InN cannot be determined from the magnetotransport experiments alone, but may be understood tentatively from the observation of the $H_{c 2}$ values of 0.3410 and $1.2 \mathrm{~T}$ being much greater than the critical magnetic field of metal indium which is $0.023 \mathrm{~T}$ (Ref. 14) suggesting that it is $\mathrm{InN}$ rather

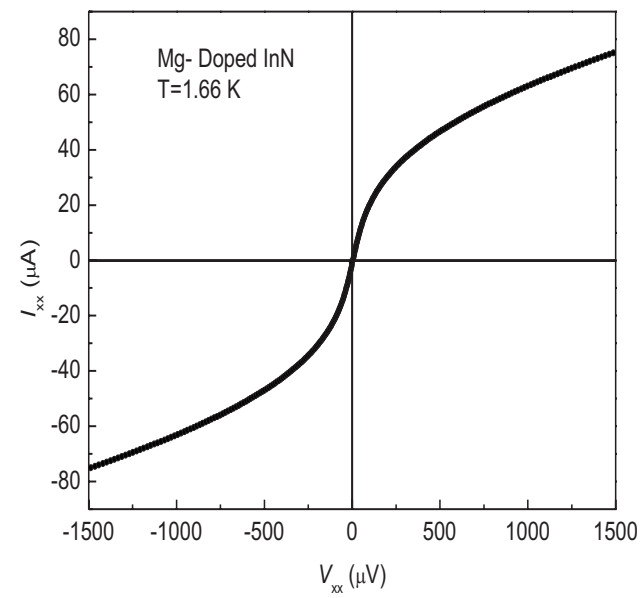

FIG. 3. Current-voltage $(I-V)$ measurements at $T=1.66 \mathrm{~K}$. 

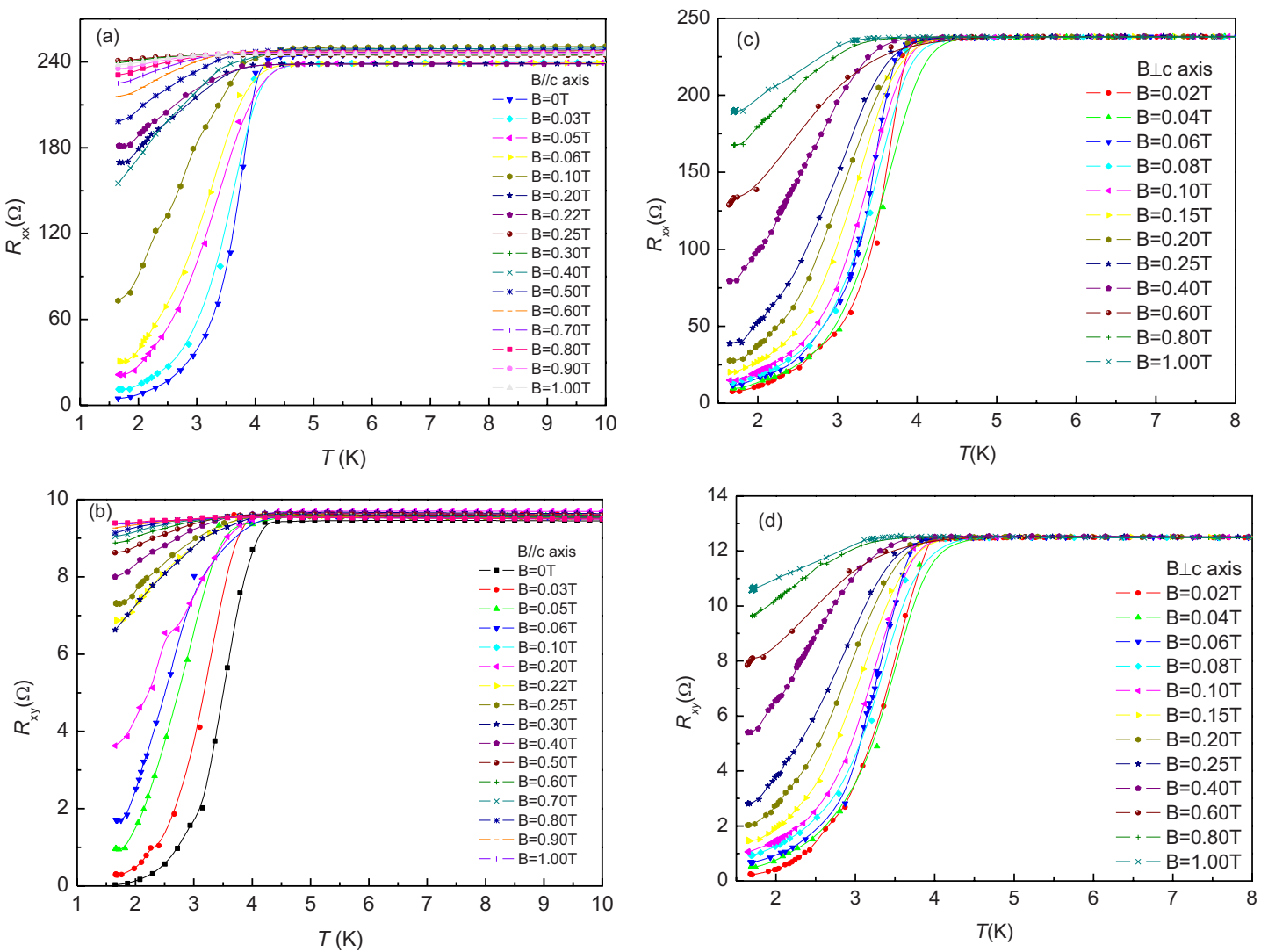

FIG. 4. (Color online) The temperature dependence of $R_{x x}$ and $R_{x y}$ at fixed magnetic field. Magnetic field direction was parallel to $c$-growth axis in (a) and (b) and perpendicular in (c) and (d).

than indium dots that become superconductive as explained above.

In Fig. 3 we show the current-voltage measurements of the Mg-doped samples at $T=1.66 \mathrm{~K}$. Up to an applied voltage of $V_{x x}<100 \mu \mathrm{V}$ we observe a behavior similar to an isolated Josephson junction (in our case it is largely smeared out due to a distribution of large number of junctions in the host matrix). At $V_{x x}>100 \mu \mathrm{V}$ current has tendency toward saturation. It is clear that at the measurement temperature $(T=1.66 \mathrm{~K})$ which is well below the transition temperature a small applied voltage of $100 \mu \mathrm{V}$ breaks the superconductivity behavior.

Another interesting observation in the temperature dependence of the resistance is the shape of the superconducting phase transition. It is clear from Fig. 4 that with increasing applied magnetic field, the shape of the $R_{x x}$ and $R_{x y}$ versus temperature curve in the superconducting state changes from convex to concave at $H_{c 2}$ values for both $B \| c$-axis and $B \perp c$-axis orientations. This observation indicates that the vortex dynamics of our InN samples are different from high-temperature superconductors. ${ }^{15}$

E.T. acknowledges the financial support of TUBITAK Ankara (Contract No. EEEAG-105E076) and Anadolu University (Contract No. 051023).

${ }^{1}$ T. L. Tansley and C. P. Foley, J. Appl. Phys. 59, 3241 (1986).

${ }^{2}$ V. Yu. Davydov, A. A. Klochikhin, R. P. Seisyan, V. V. Emtsev, S. V. Ivanov, F. Bechstedt, J. Furthmüller, H. Harima, A. V. Mudryi, J. Aderhold, O. Semchinova, and J. Graul, Phys. Status Solidi B 229, R1 (2002).

${ }^{3}$ J. Wu, W. Walukiewicz, K. M. Yu, J. W. Ager III, E. E. Haller, H. Lu, W. J. Schaff, Y. Saito, and Y. Nanishi, Appl. Phys. Lett. 80, 3967 (2002).

${ }^{4}$ T. Matsuoka, H. Okamoto, M. Nakao, H. Harima, and E. Kurimoto, Appl. Phys. Lett. 81, 1246 (2002).
${ }^{5}$ F. Bechstedt and J. Furthmuller, J. Cryst. Growth 246, 315 (2002).

${ }^{6}$ J. W. L. Yim, R. E. Jones, K. M. Yu, J. W. Ager III, W. Walukiewicz, W. J. Schaff and J. Wu, Phys. Rev. B 76, 041303 (2007).

${ }^{7}$ D. Zanato, N. Balkan, B. K. Ridley, G. Hill, and W. J. Schaff, Semicond. Sci. Technol. 19, 1024 (2004).

${ }^{8}$ E. Tiras, D. Zanato, S. Mazzucato, N. Balkan, and W. J. Schaff, Superlattices Microstruct. 36, 473 (2004).

${ }^{9}$ I. Mahboob, T. D. Veal, C. F. McConville, H. Lu, and W. J. Schaff, Phys. Rev. Lett. 92, 036804 (2004)

${ }^{10}$ I. Mahboob, T. D. Veal, L. F. J. Piper, C. F. McConville, H. Lu, W. J. Schaff, J. Furthmuller, and F. Bechstedt, Phys. Rev. B 69, 201307 (2004).

${ }^{11}$ L. F. J. Piper, T. D. Veal, I. Mahboob, C. F. McConville, H. Lu, and W. J. Schaff, Appl. Phys. Lett. 88, 252109 (2006).

${ }^{12}$ T. Inushima, V. V. Mamutin, V. A. Vekshin, S. V. Ivanov, T. Sakon, M. Motokawa, and S. Ohoya, J. Cryst. Growth 227, 481 (2001).

${ }^{13}$ K. Murano, T. Inushima, Y. Ono, T. Shiraishi, S. Ohoya, and S. Yasaka, Phys. Status Solidi B 228, 31 (2001).

${ }^{14}$ T. Inushima, N. Kato, Y. Sasaki, T. Takenobu, and M. Motokawa, Phys. Status Solidi C 2, 2271 (2005).

${ }^{15}$ T. Inushima, N. Kato, D. K. Maude, H. Lu, W. J. Schaff, R. Tauk, Y. Meziani, S. Ruffenack, O. Briot, W. Knap, B. Gil, H. Miwa, A. Yamamoto, D. Muto, Y. Nanishi, M. Higashiwaki, and T. Matsui, Phys. Status Solidi B 243, 1679 (2006).

${ }^{16}$ T. Inushima, Sci. Technol. Adv. Mater. 7, S112 (2006).

${ }^{17}$ T. Inushima, N. Kato, T. Takenobu, M. Motokawa, M. Higashiwaki, and T. Matsui, J. Phys.: Conf. Ser. 51, 279 (2006).

${ }^{18}$ T. Inushima, M. Higashiwaki, T. Matsui, T. Takenobu, and M. Motokawa, Phys. Rev. B 72, 085210 (2005).

${ }^{19}$ D. C. Ling, J. H. Cheng, Y. Y. Lo, C. H. Du, A. P. Chiu, C. A. Chang, and P. H. Chang, Phys. Status Solidi B 244, 4594 (2007).

${ }^{20}$ A. Kadir, S. Mukhopadhyay, T. Ganguli, C. Galande, M. R. Gokhale, B. M. Afora, P. Raychaudhuri, and A. Bhattacharya, Solid State Commun. 146, 361 (2008).

${ }^{21}$ H. Lu, W. J. Schaff, J. Hwang, H. Wu, W. Yeo, A. Pharkya, and L. F. Eastman, Appl. Phys. Lett. 77, 2548 (2000).

${ }^{22}$ R. E. Jones, K. M. Yu, S. X. Li, W. Walukiewicz, J. W. Ager, E. E. Haller, H. Lu, and W. J. Schaff, Phys. Rev. Lett. 96, 125505 (2006). 